\title{
Association between Contrast Sensitivity and Reading with Macular Pathology
}

Tamara Brussee, BSc ${ }^{1,2,3}$; Thomas J.T.P. van den Berg, PhD ${ }^{4}$; Ruth M.A. van Nispen, PhD ${ }^{1,2}$; Ilon de Boer, BSc ${ }^{1}$; Ger H.M.B. van Rens, MD, PhD ${ }^{1,2,3}$

${ }^{1}$ Department of Ophthalmology, VU University Medical Center, PO Box 7057, 1007 MB Amsterdam, The Netherlands; ${ }^{2}$ EMGO+ Institute for Health and Care Research, VU University Medical Center, Van der Boechorststraat 7, 1081 BT Amsterdam, The Netherlands; ${ }^{3}$ Department of Ophthalmology, Elkerliek Hospital, Wesselmanlaan 25, 5707 HA Helmond, The Netherlands; ${ }^{4}$ Netherlands Institute for Neuroscience, Royal Netherlands Academy of Arts and Sciences, Meibergdreef 47,1105 BA Amsterdam, The Netherlands.

Number of tables: 3

Numbers of figures: 4

Corresponding author:

Tamara Brussee, BSc

Department of Ophthalmology, VU University Medical Center, PO Box 7057,

1007 MB Amsterdam, the Netherlands.

Tel: +31-20-4444795.

Fax: $+31-20-4444745$

Email: t.brussee@vumc.nl

Date of submission: 8-2-2017 


\section{Abstract}

Significance: Comparison between the role of spatial and temporal contrast sensitivity in the association with reading, may provide insight into how visual tasks (such as reading) are related to primary optical or neural (or both) effects. More insight into primary visual factors influencing reading is important for understanding reading problems.

Purpose: To gain insight into the association between optical and neural components of contrast sensitivity (CS), operationalized as spatial CS (optical and neural) or temporal CS (solely neural), and reading speed in a clinical sample of participants with macular pathologies. The precision and agreement were also investigated.

Methods: The Mars test and temporal CS implementation of the C-Quant device were used to measure spatial CS and temporal CS, respectively. Tests were performed with 47 participants: mean age 77 (range 52-92) years. Associations were investigated with correlations and linear regression models. Precision was defined by coefficients of repeatability. The $95 \%$ limits of agreement between spatial CS and temporal CS values were assessed.

Results: Reading speed correlated with both spatial CS $(r=0.35, p=0.015)$ and temporal CS $(r=0.66$, $p<0.001)$. After correction for visual acuity, central loss and education level, the association between temporal CS and reading speed was not significant anymore. The coefficients of repeatability and reproducibility were 0.20 and $0.28 \log$ units (spatial CS), and 0.33 and $0.35 \log$ units (temporal CS), respectively. The values for temporal CS were 0.08 and 0.13 log units higher than for spatial CS.

Conclusions: For spatial CS and temporal CS moderate to strong correlations were found, respectively, with reading speed in patients with maculopathies. The stronger association between temporal CS and reading speed is suggested to reflect a high sensitivity for neural integrity of temporal CS. The differences in coefficients of repeatability and reproducibility could be explained by the psychometrical differences between methods.

Keywords: spatial contrast sensitivity; temporal contrast sensitivity; reading performance; reading speed; macular pathologies; retinal integrity 
The difference in luminance of a visual target on a uniform background is defined as the contrast. ${ }^{1}$ Contrast sensitivity is a function (i.e. consisting of contrast sensitivity thresholds as a function of stimulus frequencies) which is a product of optical and neural factors. ${ }^{1}$ In research and practice, two aspects of contrast sensitivity can be measured: spatial contrast sensitivity and temporal contrast sensitivity. Spatial contrast sensitivity can be measured with gratings with a sample of spatial frequencies or large letters that are presented at the peak spatial frequency. Spatial contrast sensitivity, measured with gratings or letters on a chart, is influenced by the optics of the eye, even at lower spatial frequencies. ${ }^{2}$ To measure flicker sensitivity or temporal contrast sensitivity, a flicker stimulus is presented to the eye. The concept of temporal contrast sensitivity is to better isolate neural function, as peak temporal contrast sensitivity is insensitive to optical blur. ${ }^{3}$ Since temporal contrast sensitivity is sensitive to retinal defects and insensitive to optical blur, the test is also described as a diagnostic test to detect and monitor the progression of (early) age-related macular degeneration (AMD) and to assess the efficacy of interventions. ${ }^{4,5}$

For patients with macular pathologies, besides a decrease of visual acuity (VA) also a loss of spatial and temporal contrast sensitivity is reported. ${ }^{5,6}$ Although spatial contrast sensitivity is associated with VA, it provides additional information on the aspect of visual performance. ${ }^{7}$ For patients with AMD spatial contrast sensitivity may relate better to health-related quality of life ${ }^{8}$, may associate better with utility in cost-effectiveness studies ${ }^{9}$ and may provide a broader understanding of the effects of treatment and benefits for patients. ${ }^{7}$ The main reason for visually impaired persons to seek low-vision rehabilitation is problems with reading. ${ }^{10}$ Spatial contrast sensitivity loss plays a critical role in reading, especially in persons with low vision. ${ }^{11}$ More insight into primary visual factors influencing reading performance is important for understanding a reduction in reading capacity in low vision patients with macular disease.

Comparison between the role of spatial contrast sensitivity (optical and neural) and temporal contrast sensitivity (solely neural) in the association with reading speed, may provide insight into how visual tasks (such as reading) are related to primary optical or neural (or both) effects. In an earlier study on healthy adults of various ages, a significant correlation was found between both spatial and temporal contrast sensitivity, and reading speed. ${ }^{12}$ The correlation between spatial contrast sensitivity and reading speed was found to be the stronger one and was suggested to reflect a deterioration of both optical and neural factors with increasing age. Effects are expected to be stronger in patients with 
macular pathologies, as contrast sensitivity is known to even further decrease in patients with AMD in comparison with subjects without ocular pathologies. ${ }^{13} \mathrm{~A}$ weak correlation has been reported between spatial contrast sensitivity and reading speed in patients with AMD. ${ }^{14,15}$ Sunness et al. ${ }^{16}$ found that $32 \%$ of the variability in reading speed was explained by spatial contrast sensitivity. However, to our knowledge, the association between temporal contrast sensitivity and reading speed has not yet been described for participants with macular pathologies.

Although the relevance of spatial and temporal contrast sensitivity tests is widely known and the tests have been used in research, especially temporal contrast sensitivity tests are not yet widely adopted by clinicians. The Pelli-Robson test is a method often used to measure spatial contrast sensitivity, but can be considered time-consuming or complex regarding the size of the test chart, the working distance, and the special illumination required. To overcome these issues, the Mars Letter Contrast Sensitivity Test was developed to provide a small-format portable letter contrast sensitivity chart to measure spatial contrast sensitivity at peak sensitivity. ${ }^{17}$ The Mars Letter Contrast Sensitivity Test has reasonable to good agreement with the Pelli-Robson chart, and good repeatability. ${ }^{18}$ Regarding temporal contrast sensitivity tests, although several clinical devices are available, they are not always standardized and/or are unique to each specific study. A clinical device to measure temporal contrast sensitivity was developed ${ }^{19}$ by making an adaptation to the C-Quant device ${ }^{20}$ which was originally intended to measure straylight. The temporal contrast sensitivity test measures the foveal $\left(<2^{\circ}\right)$ retinal modulation depth threshold at peak sensitivity for an $8 \mathrm{~Hz}$ homogenous large field flicker stimulus. The precision of an agreement between the two relatively new tests for measuring spatial contrast sensitivity and temporal contrast sensitivity, has been assessed. ${ }^{12}$ The coefficients of repeatability found for spatial and temporal contrast sensitivity were in agreement with previous research; the small difference between the two methods was attributed to the psychometric differences between the methods.

This study aimed to gain more insight into the primary visual factors (optical and/or neural) related to contrast sensitivity that may be important for understanding reductions in reading capacity in low vision patients with macular disease. This was also assessed previously in normally sighted subjects. ${ }^{12}$ The visual factors investigated were spatial contrast sensitivity and temporal contrast sensitivity. Because differences in individual characteristics influence the reading process, non-visual characteristics (e.g. age and education) and visual characteristics (e.g. VA, metamorphopsia, central loss and media 
condition), were taken into account. In addition, the precision of the Mars Letter Contrast Sensitivity Test to measure spatial contrast sensitivity and temporal contrast sensitivity with the C-quant device and the agreement between the two methods was assessed.

\section{Materials and methods}

\section{Participants}

Inclusion criteria were adults aged $\geq 18$ years, Dutch as the first language, and adequate cognitive skills. Cognition was screened with a validated short form of the Mini Mental State Examination. ${ }^{21}$ Also, participants were diagnosed with binocular maculopathies, the disease was stable in the last 3 months (based on patients' clinical files) and no progression was expected (based on the absence of subretinal fluid on the OCT image obtained during the first examination), and they had a stable best corrected distance VA of poorer than 0.2 LogMAR. Exclusion criteria were other eye or cognitive diseases or use of medication that could influence the results of the present study, reading disorders (e.g. dyslexia), and progression of the ocular disease. Participants were recruited in the Elkerliek Hospital, Helmond, the Netherlands. Participants who met the inclusion and exclusion criteria were invited via a letter explaining the nature/extent of the study. Informed consent was obtained from all participants.

All study procedures adhered to the Declaration of Helsinki for research involving human subjects. In addition, approval was obtained from the Institutional Review Board of the VU University Medical Center Amsterdam and the Elkerliek Hospital. Contrast sensitivity measurements were part of the test battery during an earlier study in which five Dutch continuous text reading tests were analyzed. ${ }^{22}$

Of the 201 patients approached for this study, 53 participated. Participants were significantly younger (mean: 78 years) compared with non-responders (mean: 84 years). No significant difference was found for gender. The most frequently occurring reasons for not participating were: problems with physical health, advanced age, problems with mobility/going to the hospital, and problems with mental health. Furthermore, 17 persons indicated that they did not see the benefit of participating, that they had no time to participate and/or it would be too tiring for them to participate.

\section{Materials and procedures}


All tests to measure spatial contrast sensitivity, temporal contrast sensitivity and reading speed were performed monocularly. The eye with better distance VA was selected. Refractive errors were corrected and optimal near correction was placed in a trial frame; the eye not being tested was occluded. Spatial and temporal contrast sensitivity were assessed at the preferred retinal locus for fixation, as were other tested visual tasks, such as visual acuity and reading. All measurements were performed by one experienced tester.

The Mars Letter Contrast Sensitivity Test was used to measure spatial contrast sensitivity, and the temporal contrast sensitivity test implemented within the C-Quant device was used to measure temporal contrast sensitivity. The Mars Letter Contrast Sensitivity Test is a hand-held chart with a sequence of 48 randomly ordered Sloan letters (10 different letters were used) to measure spatial contrast sensitivity at peak sensitivity. ${ }^{17}$ The Mars chart comes in three versions. The contrast range corresponds to 0.04-1.92 log contrast sensitivity, with each successive letter representing an increment of $0.04 \log$ units. Charts were presented in a randomized order and were presented on a reading stand. Chart luminance was around $90 \mathrm{~cd} / \mathrm{m}^{2}$. Participants were encouraged to guess and the testing ended when two consecutive letter-reading errors were made. Contrast sensitivity was scored using the letter-by-letter method, where a value of 0.04 log units per correct letter was given.

The temporal contrast sensitivity test measures the modulation depth threshold for an $8 \mathrm{~Hz}$ homogenous large field flicker stimulus in a two-alternative forced choice procedure; the modulation varied by 0.1 log units. The retinal projection of a large field remains virtually the same, independent of issues in the optical media such as refractive errors (aberrations) or light scattering. The stimulus field consists of a central test field with a diameter of 3.3 deg divided in two halves and projection is foveal. During the examination, the flicker stimuli were randomly presented to one of the halves of the test area with a maximum duration of $6 \mathrm{~s}$; the participant had to indicate by pressing one of two buttons, in which half of the test area the flicker was present. With the temporal contrast sensitivity test the foveal $\left(<2^{\circ}\right)$ retinal deficiencies are assessed at peak sensitivity. Details are given in the original publication. ${ }^{19}$ In the patients with central field loss, it is likely that the flickering stimulus (as well as the other stimuli used in this study) was viewed using a non-foveal preferred fixation locus. The participant held the eye to be examined close to the viewing hole of the device and the eye not being tested was covered with an occluder pad. Participants were instructed to decide which one of two fields presented flicker stimuli 
and to press the corresponding key on the device; they were encouraged to press a key even if the test fields looked the same. Tests were performed in a dimly lit room.

As also proposed earlier ${ }^{12}$ there is an essential difference between the two methods, i.e. spatial contrast sensitivity relates to a localized difference in contrast (letter on a background) and temporal contrast sensitivity relates to a periodical difference in contrast (amplitude changes back and forth over time). In accordance with generally accepted principles: i) Weber contrast, defined as ( $L_{\max }{ }^{-}$ $\left.L_{\text {min }}\right) / L_{\text {background }}$ ), was used to determine the Mars letter contrast sensitivity and ii) Michelson contrast, defined as $\left(L_{\max }-L_{\min }\right) /\left(L_{\max }+L_{\min }\right)$, was used to determine the flicker contrast sensitivity, where $L_{\max }, L_{\min }$ and $L_{\text {background }}$ are luminance maximum, minimum and background, respectively. It is important to note that, due to the different formulas used to determine contrast, there is a factor of approximately two difference between spatial contrast sensitivity and temporal contrast sensitivity, resulting in a difference of $0.3 \log$ units higher results for the temporal contrast sensitivity test.

To determine the maximum reading speed, the Radner Reading Charts ${ }^{23}$ were used. This test was chosen because reliability and other psychometric properties are known to be adequate. ${ }^{24,25}$ Two different charts were presented in randomized order; charts were presented on a reading stand. Reading distance was adjusted according to the participant's reading addition and preferred viewing distance and varied between 22 to $40 \mathrm{~cm}$; this was determined with a ruler and constantly verified during the procedure. Radner Reading Charts were read with an illumination of around $300 \mathrm{~cd} / \mathrm{m}^{2}$ but this was adjusted in a few cases if less illumination was required due to hinder from too much light reported by the participant. Reading speed was measured in standard words per minute (SWPM). ${ }^{26}$ Reading speed was determined by calculating the mean reading speed of the fastest sentence read on each chart. All testing procedures were monitored with video and audio recording; these recordings were later used to measure reading speed with a stopwatch at vocal onset. The rating of the videos was performed by three experienced and trained testers, similar to work by Radner et al. ${ }^{23}$ Distance VA was measured using a Rodenstock chart projector at a distance of 20 feet (6 m). Other visual parameters, such as ocular lens status and onset of the maculopathy, were obtained from the patient's hospital file. The Amsler grid chart was used to test for the presence of metamorphopsia and/or a central scotoma. Participants were asked, if possible, to describe and or draw the location of 
the scotoma and or appearance of the metamorphopsia. Non-visual characteristics of the participants (age and education) were asked at the start of the testing session.

The precision of the measurement can be defined as the degree to which the same method produces the same result on repeated measurements with the same individual on the same day (repeatability) and with a time interval of more days (reproducibility). ${ }^{27}$ To determine the repeatability the measurements of both the Mars Letter Contrast Sensitivity Test and the temporal contrast sensitivity test with the C-Quant device were repeated under the same conditions after 30-40 min. To determine the reproducibility of these tests, the whole testing procedure was repeated under the same conditions after a period of 2-10 weeks. Under the same conditions means that spatial contrast sensitivity and temporal contrast sensitivity were repeated in the same way regarding reading distance, illumination levels and rater, which was the same person. The optical coherence tomography (OCT) imaging technique was used to determine if there was intraretinal or subretinal fluid. If this was the case on the first examination day, patients were not invited for the second test but were referred back to their ophthalmologist. If this was the case on the second examination day, patients were withdrawn from the retest study. If changes appeared with distance VA of more than one line, the participant was withdrawn from the retest study.

\section{Statistical analysis}

To investigate the association between spatial and temporal contrast sensitivity and reading speed, the mean value of the first and second administration measurements were analyzed. In case of missing measurements, no mean could be calculated and the value of the available measurement was used. Correlations between visual characteristics (distance VA, central loss, metamorphopsia, media condition) and non-visual characteristics (age and education) were analyzed with Pearson's and Spearman's correlations. Pearson's correlation was used to evaluate the relation between continuous variables (spatial contrast sensitivity, temporal contrast sensitivity, reading speed, distance VA, age, education). Spearman correlations were used for dichotomous or ordinal variables (central loss, metamorphopsia, media condition).

Before performing linear regression analysis, for each (in)dependent variable normality and multicollinearity were checked and no issues found. Subsequently, linear regression models were fit to investigate the associations between either spatial or temporal contrast sensitivity and reading speed 
in uncorrected and corrected models. Adjustments were made for relevant visual confounders and patient characteristics.

After optimal refraction, for five participants their distance VA was better than the inclusion criteria and therefore the eye that best met the inclusion criteria was selected. In addition as a sensitivity analysis, these cases were excluded from analysis. If differences were remarkable, these were reported.

Because the visual deficit was relatively strong in several participants, not all of them could perform the temporal contrast sensitivity test. Differences between the participants who could and who could not perform the test were analyzed with independent samples t-tests and chi-square test for reading speed, visual and non-visual variables.

To determine the precision of the measurement the coefficients of repeatability and reproducibility were calculated for the spatial contrast sensitivity test and the temporal contrast sensitivity test. The mean difference and the standard deviation between two measurements were given and the distributions of these values are presented. The coefficient of repeatability or reproducibility was described as $1.96 * \mathrm{SD}$. Regarding the reliability of the temporal contrast sensitivity test, the uncertainty was assessed. The TCS test is a psychophysical test, estimating a threshold value on the basis of a number of responses. The responses can be more or less consistent. As the TCS test involves multiple stimuli/responses, and statistical analysis is based on known psychometric principles, it is possible to derive an uncertainty for each single TCS value. The mathematics of the uncertainty values are such to predict the standard deviation in case of repeated measures, and have been found to work well. ${ }^{19}$ Standard statistical tools exist to estimate uncertainty of the estimated threshold value based on the consistency of the answers and a reliability criterion of uncertainty $<0.15$ log units was considered sufficient. ${ }^{19}$ The mean uncertainty for temporal contrast sensitivity was assessed and compared with the earlier found criterion. When the uncertainty value was $>0.15$ log units, instruction to the patient may have been inadequate or misunderstood and the measurement was repeated after new instruction.

The difference between spatial contrast sensitivity and temporal contrast sensitivity was determined with the $95 \%$ limits of agreement using a Bland-Altman analysis ${ }^{28}$ and were expressed in log units. Using the limits of agreement method allows to test a proportional systematic difference between the two methods. 
All statistical analyses were performed with SPSS for Windows version 20 (SPSS IBM, New York, USA).

\section{Results}

\section{Participants}

Of the 53 participants, six were excluded from further analysis because they were unable to read any sentence on the Radner Reading Chart, or had a reading speed of $\leq 40$ SWPM. Of the remaining participants, 21 were male and 26 female. The distance acuity of the eye tested was LogMAR 0.58 ( $S D=0.26$; range 0.20-1.30). Of the remaining 47 participants, 43 had AMD, 2 had Best disease, 1 had Stargardt maculopathy, and 1 status after macular hole successfully treated with pars plana vitrectomy. Of the tested eyes, 3 had clear lenses, 16 were pseudophakic and 28 had mild to moderate cataracts. The mean number of years with maculopathies was $3(\mathrm{SD}=2$; range $1-10)$ and 18 participants had central loss. The mean number of years of education was $10(\mathrm{SD}=3$; range $6-16)$. Other vision-related patient characteristics are summarized in the bottom section of Table 1. The mean number of weeks between the first and second testing session was $4(S D=2$; range 2-10). The goal was to repeat the session within 2-6 weeks; however, although 4 participants had a longer time interval of 7-10 weeks due to personal reasons, their eye condition remained stable over that period. Four participants were not able to participate in the second testing session for the following reasons: 1 change of $\mathrm{VA}, 1$ change of OCT image, and 2 experienced the testing session as being too exhausting. In addition, there were some missing values on the variables central loss and metamorphopsia $(\mathrm{N}=4)$ and education $(\mathrm{N}=1)$.

Due to fatigue, a second measurement (on the same day) could not be performed for 3 and 2 participants for spatial and temporal contrast sensitivity, respectively. Also, 12 participants could not perform the temporal contrast sensitivity test as a whole: for 2 participants the test was not available due to technical reasons and the remaining 10 could not perform this test for various reasons (e.g. unable to see the test field, or a delayed responsiveness $>6 \mathrm{~s}$ ). Participants unable to perform the temporal contrast sensitivity test were significantly older (mean age 83 years; $p=0.019$ ) than participants (mean age 76 years) who did. For the other visual and non-visual variables no significant differences were found between the two groups. 


\section{Correlation between reading speed and contrast sensitivity}

Correlations are presented in the top section of Table 1. The average maximum reading speed was 132 SWPM (SD 53; range 44-238), spatial contrast sensitivity was 1.27 log units (SD 0.18; range 0.741.68) and temporal contrast sensitivity was $1.65 \log$ units (SD 0.39; range 0.62-2.19). Figure 1 shows the correlation between reading speed and spatial contrast sensitivity $(r=0.352 ; p=0.015)$ and temporal contrast sensitivity $(r=0.661 ; p<0.001)$. Figure 2 shows the correlation between distance VA and spatial contrast sensitivity $(r=0.287 ; p=0.051)$ and temporal contrast sensitivity $(r=0.633 ; p<0.001)$. Figure 3 shows the correlation between reading speed and distance VA $(r=0.680 ; p<0.001)$.

Not all participants could perform the temporal contrast sensitivity test due to a relatively strong visual deficit, leading to differences between the group of participants analyzed for spatial and temporal contrast sensitivity testing. Therefore, we also investigated whether the correlations between reading speed, distance VA, temporal and spatial contrast sensitivity would change if the same group of participants was used for both contrast sensitivity methods. Participants that could not perform the temporal contrast sensitivity test $(n=12)$ were removed from the analysis for spatial contrast sensitivity. The correlations were almost equal with only a slight change, e.g. the correlation between spatial contrast sensitivity and reading speed for the adjusted group was $(r=0.397, p=0.018)$.

\section{Association model for spatial contrast sensitivity or temporal contrast sensitivity and reading speed}

Based on relevant and significant correlations, we decided to adjust the regression models for distance VA, central loss and education level. Uncorrected and corrected linear regression analyses for the association between spatial contrast sensitivity or temporal contrast sensitivity and reading speed are shown in Table 2. The uncorrected analyses showed a significant association between spatial contrast sensitivity and reading speed $(b=104 ; p=0.015)$ and between temporal contrast sensitivity and reading speed $(b=88 ; p<0.001)$. Reading speed increased with increasing spatial and temporal contrast sensitivity. In the uncorrected model spatial contrast sensitivity explained $12 \%$ of the variance and temporal contrast sensitivity explained $44 \%$ of the variance. The corrected models 
explained $64 \%$ and $61 \%$ of the variance for respectively spatial contrast sensitivity and temporal contrast sensitivity in the association with reading speed. However, the association between temporal contrast sensitivity and reading speed was not significant anymore.

In addition, a sensitivity analysis was performed. Five participants were excluded from the analysis because, after optimal refraction, their distance VA was better than the inclusion criterion. When removed from the corrected model, spatial contrast sensitivity $(b=71 ; p=0.073)$ significance level slightly dropped and was on the verge of significance in association with reading speed. The association between temporal contrast sensitivity and reading did not change significantly, only the regression coefficients slightly changed.

\section{Precision of the spatial/temporal contrast sensitivity test and comparison between tests}

In Figure 4 (for each test separately) the difference between the two administrations, with the longer time interval, is plotted as a function of the mean of spatial contrast sensitivity or temporal contrast sensitivity. Table 3 presents the mean differences, SDs and coefficient of repeatability and reproducibility. The average value for the first minus second administration measurement on the same day for spatial contrast sensitivity was $-0.01 \log$ units and for temporal contrast sensitivity was 0.06 log units. The mean uncertainty for temporal contrast sensitivity was 0.11 (SD 0.03 and SD 0.04) log units for the first and second measurement on the same day, respectively, and 0.11 (SD 0.02) log units for the measurement a few weeks later; all these values are below the uncertainty reliability criterion of $<0.15 \log$ units. The coefficients of repeatability and reproducibility were 0.20 and 0.28 log units, respectively, for spatial contrast sensitivity; and 0.33 and 0.35 log units, respectively, for temporal contrast sensitivity.

Table 3 presents the agreement between spatial and temporal contrast sensitivity. As explained earlier, ${ }^{12}$ due to the different formulas used to determine spatial and temporal contrast sensitivity, a correction factor of two (difference 0.3 log units) needs to be applied to determine the difference between the two methods. After applying the correction factor, the values for the temporal contrast sensitivity were 0.08 and 0.13 log units (first and second measurement, respectively) higher than those for the spatial contrast sensitivity. 


\section{Discussion}

The aim of this study was to determine to what extent optical versus neural aspects influence reading speed for individuals with macular pathologies, and to assess the precision and agreement of two relatively new tests for measuring spatial and temporal contrast sensitivity in this group.

Spatial contrast sensitivity can be considered the combined result of neural sensitivity and optical factors and, as such, includes temporal contrast sensitivity (considered to be solely neural sensitivity). In the present study, both spatial and temporal contrast sensitivity were tested in participants with maculopathies, allowing to compare both methods and their association with reading speed in this group. Results of the correlation between spatial contrast sensitivity and reading speed are in accordance with earlier research. ${ }^{11,15,29,30}$ To our knowledge the association between temporal contrast sensitivity and reading speed has not earlier been described for participants with macular pathologies. In the present study, moderate $(r=0.352 ; p=0.015)$ to strong $(r=0.661 ; p<0.001)$ correlations were found between spatial contrast sensitivity and temporal contrast sensitivity, respectively, and reading speed. After correction for confounding in the linear regression model by distance VA, central loss and education level, the association between temporal contrast sensitivity and reading speed was not significant anymore.

The stronger association found between temporal contrast sensitivity and reading speed, tested in participants with maculopathies, is suggested to reflect a high sensitivity for neural integrity of the temporal contrast sensitivity test. As also proposed by others, temporal contrast sensitivity ${ }^{31}$ as well as reading speed ${ }^{32}$ are relatively unaffected (or less affected) by media opacity and can therefore be used as a potential vision test to assess neural integrity. One could speculate about an explanation for the high sensitivity of temporal contrast sensitivity tests for neural integrity: It is possible that spatial and temporal contrast sensitivity target different retinal mechanisms. Flicker stimulation (in comparison to a static stimulation) is reported to lead to a higher metabolic demand of retinal tissue (vasodilatation, ${ }^{33}$ increased blood flow, ${ }^{34}$ and oxygen levels ${ }^{35}$ ). In eyes with AMD, this specific mechanism may be affected allowing flicker stimuli to detect functional retinal deficiencies. ${ }^{13,36,37}$ 
Certain visual characteristics and non-visual characteristics that influence the association between spatial or temporal contrast sensitivity and reading speed were found. The association between temporal contrast sensitivity and reading speed was not significant anymore after correction for in particular distance VA. Therefore, we cannot rule out that other visual functioning tests or patient characteristics mask this association in a sample of patients with progressive AMD.

It can be questioned to what extent distance VA can explain or predict deficits in reading speed in patients with maculopathies. ${ }^{38}$ Other factors not measured in the present study, such as eccentric viewing, fixation stability, reduced visual span, and impaired eye movement control are known to influence the reading process. $^{39-42}$ Furthermore, especially in the case for early AMD where subtle alterations in the macula appear before VA decreases, other visual function tests, in particular spatial and temporal contrast sensitivity, visual adaptation, central visual field and colour discrimination have been reported to reflect the functional status of the macula better than VA. ${ }^{13}$ This in contrast to the present study where most patients had advanced geographic atrophy, which had a significant impact on distance $V A$ and reading speed.

It is therefore interesting for future research to test temporal contrast sensitivity and the relation with reading speed for patients with early maculopathies and with adequate distance VA. It was also suggested in the literature that functional assessment of retinal integrity and retinal changes, in combination with imaging techniques and clinical grading, could have a high potential for monitoring and diagnosing AMD. 5, 43, 44 It would be interesting to study whether the short-duration temporal contrast sensitivity test used in the present study could serve this purpose.

In the present study, the correlation found between temporal contrast sensitivity and reading ( $r=0.661$; $p<0.001)$ was higher than in an earlier study in participants without macular pathologies $(r=0.258$; $p=0.042)$. This might be explained by the larger variability in macular function in the present study, which seems to be strongly indicated by the temporal contrast sensitivity test, being a selective neural test. The correlation found between spatial contrast and reading speed $(r=0.352 ; p=0.015)$ is very similar to the association found in another study with participants with no ocular pathologies $(r=0.470$; $\mathrm{p}=<0.001) .{ }^{12}$ The smaller age range in the present study (52-92 years) in comparison with the group without ocular pathologies (18-86 years), probably explains the lower and non significant correlations between age and spatial or temporal contrast sensitivity in comparison with the results found in the 
latter study. ${ }^{12}$ Also the stronger source of variation (macular pathology) might causes the weaker source of variation (age) to be less significant.

Regarding the feasibility of the two contrast sensitivity tests, of the 47 participants analyzed 10 could not perform the temporal contrast sensitivity test due to difficulty in seeing the test field, perceiving the flicker or they had a delay in their responsiveness and could not react within the $6 \mathrm{~s}$ of the stimulus duration. These 10 participants were significantly older than the participants who could perform the temporal contrast sensitivity test. The differences between the two groups on the other variables (e.g. distance VA, central loss, metamorphopsia, media condition and education) was not significant. It is unclear how the fact that the temporal contrast sensitivity test could not be performed by all participants may have influenced the association found between temporal contrast sensitivity and reading speed. We looked into how the correlations between reading speed and spatial contrast sensitivity would change if the group of participants who could not perform the temporal contrast sensitivity test were excluded from the analysis, we found that the correlation changed only slightly.

Considering the precision of the tests at the first administration measurement and at the retest, the coefficient of repeatability and reproducibility was 0.22 and 0.28 log units for the spatial contrast sensitivity test and was 0.33 and 0.35 log units for the temporal contrast sensitivity test, respectively. The difference in coefficient of repeatability and reproducibility values between the two methods might be attributed to the psychometric differences between the methods. The spatial contrast sensitivity test (which uses 10 Sloan letters) has a 10-alternative forced choice procedure compared with a twoalternative forced choice procedure of the temporal contrast sensitivity test. The coefficient of reproducibility for spatial contrast sensitivity is in agreement with that of Haymes et al. ${ }^{45}$ These authors found a coefficient of reproducibility of 0.24 log units for a similar group of patients (only slightly younger and with worsened VA). Dougherty et al. ${ }^{46}$ found a slightly lower coefficient of repeatability of $0.20 \log$ units for a group of participants about 20 years younger (mean age 57 years). Both studies used the Mars letter Contrast Sensitivity Test to measure spatial contrast sensitivity. The present study is the first to describe the repeatability and reproducibility of temporal contrast sensitivity measured with the adaptation to the C-Quant device for a group of participants with maculopathies.

The agreement between the spatial and temporal contrast sensitivity methods was similar to an earlier study (including normally sighted persons) in which the values of temporal contrast sensitivity were 0.1 
log units higher than those of spatial contrast sensitivity. ${ }^{12}$ As mentioned, this difference might be attributed to optical mechanisms leading to a worsened image on the retina for the spatial (specifically, letter) contrast sensitivity, and to a difference between the neurons in the parvo-cellular and magnocellular pathways mediating (primarily) the spatial contrast sensitivity and temporal contrast sensitivity, respectively.

In the present study, the lack of clearly-defined size and location of macular scotomas is a limitation; however, to address this problem, spatial contrast sensitivity, temporal contrast sensitivity, reading speed and the Amsler chart were assessed monocularly. To assess all tests monocularly, it was assumed that the preferred retinal locus of the participants would be the same for the different tests, as no shift could be made to the fellow eye. However, due to possibly unstable fixation, this cannot be guaranteed. ${ }^{41}$ Furthermore, a significant proportion of patients with central loss will not be identified using the Amsler chart alone and more advanced devices are needed. ${ }^{47}$ The use of micro-perimeters, such as the Nidek MP-1 and Haag-Streit Maia which includes a scanning laser ophthalmoscope (SLO), might provide more insight into the relation between contrast sensitivity and reading, and the influence of macular problems. ${ }^{48}$ Furthermore, although all our participants had atrophic areas within the limits of accuracy subjectively assessed, the size could not be accurately measured with the OCT (also due to unstable fixation). Of importance is that contrast thresholds were determined at peak spatial frequency, instead of a whole contrast sensitivity function curve; this might also be considered a study limitation.

\section{Conclusion}

The aims of this study were to gain insight into the extent in which optical versus neural aspects influence reading speed for individuals with macular pathologies, and to assess the precision and agreement of two relatively new tests for measuring spatial and temporal contrast sensitivity. For spatial contrast sensitivity and temporal contrast sensitivity, moderate to strong correlations were found, respectively, with reading speed. The stronger association found between temporal contrast sensitivity and reading speed is suggested to reflect a high sensitivity for neural integrity of the temporal contrast sensitivity test. However, we cannot rule out that other visual functioning tests or patient characteristics mask the association in a sample of patients with progressive AMD. The coefficients of repeatability and reproducibility for spatial contrast sensitivity found in the present study 
were better in comparison with the temporal contrast sensitivity, which might be attributed to the psychometric differences between the methods. The coefficients of repeatability and reproducibility for spatial contrast sensitivity found in the present study are found to be good and in agreement with previous research. A downside of the temporal contrast sensitivity test is that not all participants could manage to perform the test because of profound vision loss. However, the temporal contrast sensitivity test might have a high potential for diagnosing early AMD and monitoring of patients in clinical practice.

\section{Acknowledgments}

This study received grants from the SNOO (Foundation for Dutch Ophthalmologic Research) and the Elkerliek Hospital (Helmond, the Netherlands).

The authors report no conflicts of interest and have no proprietary interest in any of the materials. The Royal Netherlands Academy of Arts and Sciences owns a patent on straylight measurement, which it licenses to Oculus $\mathrm{GmbH}$ for the C-Quant instrument. 


\section{REFERENCES}

1. Pelli DG, Bex P. Measuring contrast sensitivity. Vision Res 2013;90:10-14.

2. Woods RL, Strang NC, Atchison DA. Measuring contrast sensitivity with inappropriate optical correction. Ophthalmic Physiol Opt 2000;20:442-451.

3. Vianya-Estopa M, Douthwaite WA, Noble BA, Elliott DB. Capabilities of potential vision test measurements: clinical evaluation in the presence of cataract or macular disease. J Cataract Refract Surg 2006;32:1151-1160.

4. Dimitrov PN, Robman LD, Varsamidis M, et al. Visual function tests as potential biomarkers in age-related macular degeneration. Invest Ophthalmol Vis Sci 2011;52:9457-9469.

5. Mayer MJ, Ward B, Klein R, Talcott JB, Dougherty RF, Glucs A. Flicker sensitivity and fundus appearance in pre-exudative age-related maculopathy. Invest Ophthalmol Vis Sci 1994;35:1138-1149.

6. Bellmann C, Unnebrink K, Rubin GS, Miller D, Holz FG. Visual acuity and contrast sensitivity in patients with neovascular age-related macular degeneration. Results from the Radiation Therapy for Age-Related Macular Degeneration (RAD-) Study. Graefes Arch Clin Exp Ophthalmol 2003;241:968-974.

7. Mones J, Rubin GS. Contrast sensitivity as an outcome measure in patients with subfoveal choroidal neovascularisation due to age-related macular degeneration. Eye (Lond) 2005;19:1142-1150.

8. Bansback N, Czoski-Murray C, Carlton J, et al. Determinants of health related quality of life and health state utility in patients with age related macular degeneration: the association of contrast sensitivity and visual acuity. Qual Life Res 2007;16:533-543.

9. Butt T, Patel PJ, Tufail A, Rubin GS. Modelling cost effectiveness in neovascular age-related macular degeneration: the impact of using contrast sensitivity vs. visual acuity. Appl Health Econ Health Policy 2014;12:289-297.

10. Brown JC, Goldstein JE, Chan TL, Massof R, Ramulu P. Characterizing functional complaints in patients seeking outpatient low-vision services in the United States. Ophthalmology 2014;121:1655-1662.

11. Giacomelli G, Virgili G, Giansanti F, et al. Clinical and microperimetric predictors of reading speed in low vision patients: a structural equation modeling approach. Invest Ophthalmol Vis Sci 2013;54:4403-4408.

12. Brussee T, van den Berg TJ, van Nispen RM, van Rens GH. Associations Between Spatial and Temporal Contrast Sensitivity and Reading. Optom Vis Sci 2017;94:329-338.

13. Neelam K, Nolan J, Chakravarthy U, Beatty S. Psychophysical function in age-related maculopathy. Surv Ophthalmol 2009;54:167-210.

14. Bullimore MA, Bailey IL. Reading and eye movements in age-related maculopathy. Optom Vis Sci 1995;72:125-138. 
15. Cacho I, Dickinson CM, Smith HJ, Harper RA. Clinical impairment measures and reading performance in a large age-related macular degeneration group. Optom Vis Sci 2010;87:344349.

16. Sunness JS, Rubin GS, Applegate CA, et al. Visual function abnormalities and prognosis in eyes with age-related geographic atrophy of the macula and good visual acuity. Ophthalmology 1997;104:1677-1691.

17. Arditi A. Improving the design of the letter contrast sensitivity test. Invest Ophthalmol Vis Sci 2005;46:2225-2229.

18. Thayaparan K, Crossland MD, Rubin GS. Clinical assessment of two new contrast sensitivity charts. Br J Ophthalmol 2007;91:749-752.

19. van den Berg TJ, Franssen L, Kruijt B, Coppens JE. Psychophysics, reliability, and norm values for temporal contrast sensitivity implemented on the two alternative forced choice C-Quant device. J Biomed Opt 2011;16:085004.

20. Franssen L, Coppens JE, van den Berg TJ. Compensation comparison method for assessment of retinal straylight. Invest Ophthalmol Vis Sci 2006;47:768-776.

21. Callahan CM, Unverzagt FW, Hui SL, Perkins AJ, Hendrie HC. Six-item screener to identify cognitive impairment among potential subjects for clinical research. Med Care 2002;40:771781.

22. Brussee $T$, van Nispen RMA, Alagahgi B, van Rens GHMB. Reliability of five continuous text reading performance tests in participants with maculopathies. (In press)

23. Radner W, Obermayer W, Richter-Mueksch S, Willinger U, Velikay-Parel M, Eisenwort B. The validity and reliability of short German sentences for measuring reading speed. Graefes Arch Clin Exp Ophthalmol 2002;240:461-467.

24. Brussee T, van Nispen RM, Klerkx EM, Knol DL, van Rens GH. Comparison of reading performance tests concerning difficulty of sentences and paragraphs and their reliability. Ophthalmic Physiol Opt 2015;35:324-335.

25. Burggraaff MC, van Nispen RM, Hoek S, Knol DL, van Rens GH. Feasibility of the Radner Reading Charts in low-vision patients. Graefes Arch Clin Exp Ophthalmol 2010;248:16311637.

26. Carver RP. Reading Rate: A Review of Research and Theory San Diego, CA: Academic Press; 1990.

27. McAlinden C, Khadka J, Pesudovs K. Statistical methods for conducting agreement (comparison of clinical tests) and precision (repeatability or reproducibility) studies in optometry and ophthalmology. Ophthalmic Physiol Opt 2011;31:330-338.

28. Bland JM, Altman DG. Measuring agreement in method comparison studies. Statistical Methods in Medical Research 1999;8:135-160.

29. Amore FM, Silvestri V, Turco S, De RF, Cruciani F. Rehabilitative approach in patients with ring scotoma. Can J Ophthalmol 2013;48:420-426. 
30. McClure ME, Hart PM, Jackson AJ, Stevenson MR, Chakravarthy U. Macular degeneration: do conventional measurements of impaired visual function equate with visual disability? $\mathrm{Br} \mathrm{J}$ Ophthalmol 2000;84:244-250.

31. del Romo GB, Douthwaite WA, Elliott DB. Critical flicker frequency as a potential vision technique in the presence of cataracts. Invest Ophthalmol Vis Sci 2005;46:1107-1112.

32. Pesudovs K, Patel B, Bradbury JA, Elliott DB. Reading speed test for potential central vision measurement. Clin Experiment Ophthalmol 2002;30:183-186.

33. Formaz F, Riva CE, Geiser M. Diffuse luminance flicker increases retinal vessel diameter in humans. Curr Eye Res 1997;16:1252-1257.

34. Garhofer G, Zawinka C, Resch H, Huemer KH, Dorner GT, Schmetterer L. Diffuse luminance flicker increases blood flow in major retinal arteries and veins. Vision Res 2004;44:833-838.

35. Shakoor A, Blair NP, Mori M, Shahidi M. Chorioretinal vascular oxygen tension changes in response to light flicker. Invest Ophthalmol Vis Sci 2006;47:4962-4965.

36. Feigl B, Brown B, Lovie-Kitchin J, Swann P. Functional loss in early age-related maculopathy: the ischaemia postreceptoral hypothesis. Eye (Lond) 2007;21:689-696.

37. Phipps JA, Dang TM, Vingrys AJ, Guymer RH. Flicker perimetry losses in age-related macular degeneration. Invest Ophthalmol Vis Sci 2004;45:3355-3360.

38. Crossland MD, Culham LE, Rubin GS. Predicting reading fluency in patients with macular disease. Optom Vis Sci 2005;82:11-17.

39. Legge GE, Ahn SJ, Klitz TS, Luebker A. Psychophysics of reading--XVI. The visual span in normal and low vision. Vision Res 1997;37:1999-2010.

40. Calabrese A, Bernard JB, Faure G, Hoffart L, Castet E. Eye movements and reading speed in macular disease: the shrinking perceptual span hypothesis requires and is supported by a mediation analysis. Invest Ophthalmol Vis Sci 2014;55:3638-3645.

41. Crossland MD, Culham LE, Rubin GS. Fixation stability and reading speed in patients with newly developed macular disease. Ophthalmic Physiol Opt 2004;24:327-333.

42. Rubin GS, Freely $M$. The role of eye movements during reading in patients with age-related macular degeneration (AMD). In: 33 ed.; 2009:120-126.

43. Dimitrov PN, Robman LD, Varsamidis M, et al. Relationship between clinical macular changes and retinal function in age-related macular degeneration. Invest Ophthalmol Vis Sci 2012;53:5213-5220.

44. Luu CD, Dimitrov PN, Robman L, et al. Role of flicker perimetry in predicting onset of latestage age-related macular degeneration. Arch Ophthalmol 2012;130:690-699.

45. Haymes SA, Roberts KF, Cruess AF, et al. The letter contrast sensitivity test: clinical evaluation of a new design. Invest Ophthalmol Vis Sci 2006;47:2739-2745.

46. Dougherty BE, Flom RE, Bullimore MA. An evaluation of the Mars Letter Contrast Sensitivity Test. Optom Vis Sci 2005;82:970-975. 
47. Crossland M, Rubin G. The Amsler chart: absence of evidence is not evidence of absence. $\mathrm{Br} \mathrm{J}$ Ophthalmol 2007;91:391-393.

48. Calabrese A, Bernard JB, Hoffart L, et al. Wet versus dry age-related macular degeneration in patients with central field loss: different effects on maximum reading speed. Invest Ophthalmol Vis Sci 2011;52:2417-2424. 
Table. 1. Pearson's and Spearman's correlation coefficients of potential covariates with SWPM for the Radner sentences and participants' characteristics (mean values).

\begin{tabular}{|c|c|c|c|c|c|c|c|c|c|}
\hline & $\begin{array}{l}\text { Reading } \\
\text { speed } \\
\text { (SWPM) }\end{array}$ & $\begin{array}{l}\text { SCS } \\
\text { (log units) }\end{array}$ & $\begin{array}{l}\text { TCS } \\
\text { (log units) }\end{array}$ & $\begin{array}{l}\text { Distance } \\
\text { VA } \\
\text { (logMar) }\end{array}$ & $\begin{array}{l}\text { Central } \\
\text { loss } \\
\text { (yes/no) }\end{array}$ & $\begin{array}{l}\text { Metamor- } \\
\text { phopsia } \\
\text { (yes/no) }\end{array}$ & $\begin{array}{l}\text { Media } \\
\text { condition } \\
\text { (clearl } \\
\text { unclear) }\end{array}$ & $\begin{array}{l}\text { Age } \\
\text { (years) }\end{array}$ & $\begin{array}{l}\text { Education } \\
\text { (years) }\end{array}$ \\
\hline $\operatorname{SCS}(\mathrm{N}=47)$ & $0.352^{*} \dagger$ & & & & & & & & \\
\hline $\operatorname{TCS}(\mathrm{N}=35)$ & $0.661^{* *} \dagger$ & $0.603^{* *} \dagger$ & & & & & & & \\
\hline Distance VA & $-0.680^{* *} \dagger$ & $-0.287 \dagger$ & $-0.633^{* *} \dagger$ & & & & & & \\
\hline Central loss & $-0.582^{* *} \ddagger$ & $-0.047 \ddagger$ & $-0.507^{* *} \ddagger$ & $0.462^{* *} \ddagger$ & & & & & \\
\hline $\begin{array}{l}\text { Metamor- } \\
\text { phopsia }\end{array}$ & $-0.107 \ddagger$ & $0.039 \ddagger$ & $-0.102 \ddagger$ & $0.058 \ddagger$ & $0.341^{*} \ddagger$ & & & & \\
\hline $\begin{array}{l}\text { Media } \\
\text { condition }\end{array}$ & $-0.003 \ddagger$ & $0.108 \ddagger$ & $0.026 \ddagger$ & $0.025 \ddagger$ & $0.099 \ddagger$ & $0.096 \ddagger$ & & & \\
\hline Age & $0.030 \dagger$ & $-0.011 \dagger$ & $0.279 \dagger$ & $-0.234 \dagger$ & $-0.266 \ddagger$ & $-0.154 \ddagger$ & $-0.131 \ddagger$ & & \\
\hline Education & $-0.323^{*} \dagger$ & $0.267 \dagger$ & $-0.117 \dagger$ & $0.141 \dagger$ & $0.242 \ddagger$ & $0.106 \ddagger$ & $0.082 \ddagger$ & $-0.145 \dagger$ & \\
\hline Mean (SD) & $132(53)$ & $1.27(0.18)$ & $1.65(0.39)$ & $0.58(0.26)$ & $38 \%$ yes & $68 \%$ yes & $36 \%$ Clear & 77 (8) & $10(3)$ \\
\hline range & 44 to 238 & $\begin{array}{r}0.74 \text { to } \\
1.68\end{array}$ & $\begin{array}{r}0.62 \text { to } \\
2.19\end{array}$ & $\begin{array}{r}1.30 \text { to } \\
0.20\end{array}$ & & & & 52 to 92 & 6 to16 \\
\hline
\end{tabular}

Table 1. **Correlations are significant at the 0.001 level (2-tailed), *Correlations are significant at the 0.05 level (2-tailed). SWPM; standard words per minute, VA; visual acuity. SCS; spatial contrast sensitivity, TCS; temporal contrast sensitivity. †Pearson's correlation was used to evaluate the relation between two continuous variables. $¥$ Spearman correlation was used to evaluate the monotonic relation between two continuous or ordinal variables. Ordinal variables were; central loss, metamorphopsia, media condition, sex. 
Table 2. Crude and adjusted models of the estimated associations between spatial or temporal contrast sensitivity and reading speed (in SWPM).

\begin{tabular}{|c|c|c|c|c|c|}
\hline & $\begin{array}{r}\text { Crude model } \\
\mathrm{b}[95 \%-\mathrm{Cl}]\end{array}$ & $p$ & $\begin{array}{r}\text { Adjusted model } \\
\text { b [95\%-Cl] }\end{array}$ & $p$ & \\
\hline $\operatorname{SCS}(\mathrm{N}=47)$ & $104.2[20.9 ; 187.4]$ & 0.015 & $76.0[6.8 ; 145.3]$ & 0.032 & \\
\hline Distance VA & & & $-91.5[-139.4 ;-43.6]$ & $<0.001$ & \\
\hline Central loss & & & $-29.7[-54.7 ;-4.8]$ & 0.021 & \\
\hline Education & & & $-4.1[-8.8 ; 0.5]$ & 0.082 & \\
\hline $\operatorname{TCS}(\mathrm{N}=35)$ & $88.3[52.9 ; 123.8]$ & $<0.001$ & $31.2[-22.5 ; 85.0]$ & 0.243 & \\
\hline Distance VA & & & $-84.6[-160.5 ;-8.7]$ & 0.030 & \\
\hline Central loss & & & $-24.9[-57.3 ; 7.4]$ & 0.125 & \\
\hline Education & & & $-2.7[-8.9 ; 3.5]$ & 0.375 & \\
\hline
\end{tabular}


Table 3. Precision of the spatial and temporal contrast sensitivity test and difference in values between the two methods.

\begin{tabular}{|c|c|c|c|c|c|}
\hline & $\begin{array}{l}\text { CS values (log) test } 1 \\
\text { Mean (SD) }\end{array}$ & $\begin{array}{l}\text { CS values (log) test } 2 \\
\text { Mean (SD) }\end{array}$ & $\begin{array}{l}\Delta \text { CS values (log) } \\
\text { Mean (SD) }\end{array}$ & $\begin{array}{l}\text { Coefficient of repeatabilityl } \\
\text { reproducibility }\end{array}$ & $\begin{array}{l}\text { (95\% limits of } \\
\text { agreement) }\end{array}$ \\
\hline SCS test, measurement 1 - 2 same day $(\mathrm{N}=43)$ & $1.26(0.19)$ & $1.26(0.17)$ & $-0.01(0.10)$ & 0.20 & \\
\hline TCS test, measurement $1-2$ same day ( $N=33$ ) & $1.62(0.40)$ & $1.69(0.38)$ & $0.06(0.17)$ & 0.33 & \\
\hline $\begin{array}{l}\text { SCS test, measurement } 1 \text { - } 2 \text { time interval 2- } 10 \\
\text { weeks }\end{array}$ & $1.26(0.19)$ & $1.27(0.20)$ & $0.00(0.15)$ & 0.28 & \\
\hline $\begin{array}{l}\text { TCS test, measurement } 1 \text { - } 2 \text { time interval 2- } 10 \\
\text { weeks }\end{array}$ & $1.62(0.40)$ & $1.72(0.36)$ & $0.07(0.18)$ & 0.35 & \\
\hline \multirow{2}{*}{$\begin{array}{l}\text { TCS test - SCS test, for administration } \\
\text { measurement } 1 \text { and } 2 \text { on the same day }\end{array}$} & & & $0.38+(0.36)$ & & {$[-0.31 ; 1.08] \dagger$} \\
\hline & & & $0.43 \ddagger(0.29)$ & & {$[-0.15 ; 1.00] \ddagger$} \\
\hline
\end{tabular}

Table 3. SCS; spatial contrast sensitivity, TCS; temporal contrast sensitivity, $r$ : Lin's concordance correlation coefficients were used for the correlation between two administration measurements for the spatial CS and temporal CS test. Pearson correlation coefficients were used for the correlation between the spatial CS and temporal CS test, ${ }^{*}$ Correlation is significant at the 0.01 level (2-tailed). $\dagger$ : first administration measurement, $\neq$ : second administration measurement. $\Delta$ difference between two administration measurements or between two methods. Values are in log units. 
Figure 1. Association between spatial and temporal contrast sensitivity and reading speed. R; Pearson's correlation coefficients, ${ }^{* *}$ correlation is significant at the 0.01 level (2-tailed), ${ }^{*}$ correlation is significant at the 0.05 level (2-tailed). SCS; spatial contrast sensitivity, TCS; temporal contrast sensitivity, RS; reading speed.

Figure 2. Association between spatial or temporal contrast sensitivity and distance visual acuity. R; Pearson's correlation coefficients, ${ }^{\star *}$ correlation is significant at the 0.01 level (2-tailed). SCS spatial contrast sensitivity, TCS; temporal contrast sensitivity, VA; visual acuity.

Figure 3. Association between reading speed and distance visual acuity. R; Pearson's correlation coefficients, ${ }^{* *}$ correlation is significant at the 0.01 level (2-tailed)

Figure 4. Reproducibility of the spatial (A) and temporal contrast sensitivity (B) tests, as judged from the difference between the two administration measurements with a time interval of 2-10 weeks. SCS; spatial contrast sensitivity, TCS; temporal contrast sensitivity 